\title{
On Weak Well-posedness of Mixed Problems for Hyperbolic Sy:tems
}

\author{
By \\ Koji KaSAHARA*
}

1. Introduction. In this paper, we treat a mixed problem for first order hyperbolic systems of partial differential equations with constant coefficients in quarter space and show a necessary and sufficient condition for the weak $L^{2}$-wellposedness of mixed problems. For equations hyperbolic in Gårding's sense, the mixed problem has been investigated by Hersh ([1] and $[2]$ ), stating that the condition that the Lopatinski determinant of the problem is not equal to zero (in this paper this is represented in the form (3)) is equivalent to the weak $L^{2}$-wellposedness. But his proof is incomplete by the following reasons. First the generalized eigenvectors $w_{j}$ are used in the construction of the elementary solution $\widehat{U}(\tau)$ (in [2]; also in [1] we refer to $v$ ), but these $w_{j}$ were only constructed in pointwise sense, hence, if the multiplicity of eigenvalues changes, the construction must also be changed. Thus the smoothness of the construction of $w_{j}$ with respect to the variables $\tau$ and $\eta$ should have been shown. Secondly, to show that the elementary solution increases at most by polynomial order as $|\tau|^{2}+|\eta|^{2} \rightarrow+\infty$, the explicit formula of the generalized eigenvectors $w_{j}$, could not be used, since the formula itself depends on $(\tau, \eta)$.

We shall here show that these difficulties can be overcome by using Seidenberg-Tarski elimination theorem and the Cauchy integral formula for matrices and vectors.

2. We consider a system of first order linear partial differential equations with constant coefficients

Received October 29, 1970.

Communicated by S. Matsuura.

* Mathematical Institute, Faculty of Liberal Arts. Kyoto Lniversity. 


$$
\frac{\partial u}{\partial t}=A_{0} \frac{\partial u}{\partial x}+\sum_{j=1}^{m} A_{j} \frac{\partial u}{\partial y_{j}}+C u
$$

where $u=u(t, x, y)$ is an $n$-dimensional vector-valued function, and $A_{j}$ $(j=0,1, \cdots, m)$ are $n \times n$ square matrices. We assume that this system is hyperbolic in Garding's serse, that is, the real part of the roots $\tau$ of the equation

$$
\operatorname{det}\left(\tau I-A_{0} i \xi-\sum_{j=1}^{m} A_{j} i \eta_{j}-C\right)=0
$$

are bounded as $\xi$ and $\eta_{j}$ run over the real field. We denote this bound by $M_{0}$. Moreover, we assume that the matrix $A_{0}$ is non-singular, which means that the hyperplane $x=0$ is not characteristic. By virtue of hyperbolicity, eigenvalues of $A_{0}$ are real. Let the number of negative eigenvalues of $A_{0}$ be $k$. Besides, a boundary matrix $B$ is also considered in order to set a homogeneous boundary condition $B u(t, 0, y)=0$ on $x=0$. We denote by $R_{+}^{m+1}$ the half space $\{(t, x, y) ; t=0, x \geqslant 0\}$ and by $R_{*}^{m+2}$ the quarter space $\{(t, x, y) ; t \geqslant 0, x \geqslant 0\}$. We say that the mixed problem is weakly $L^{2}$-well-posed, if there exist a real number $\nu$ such that for any given real number $\mu$ and any initial function $u_{0}(x, y)$ given in the space $H^{\mu}\left(R_{+}^{m+1}\right)$ there exist a solution of (1) in the space $\widetilde{H}^{\mu-\nu}\left(R_{*}^{m+2}\right)=\left\{u: e^{-\sigma t} u \in H^{\mu-\nu}\left(R_{*}^{m+2}\right)\right\}\left(\sigma>M_{0}^{2}\right)$ with the initial condition

$$
u(0, x, y)=u_{0}(x, y) \quad \text { for } \quad x \geqslant 0
$$

and the boundary condition

$$
B u(t, 0, y)=0 \quad \text { for } \quad t \geqslant 0 .
$$

Our purpose in this paper is to formulate a necessary and sufficient condition to be satisfied by the boundary matrix $B$ in order that the mixed problem (1) and (2) is weakly $L^{2}$-well-posed. By changing the representation in (1), we get

$$
\frac{\partial u}{\partial x}=A_{0}^{-1}\left(\frac{\partial}{\partial t}-\sum_{j=1}^{m} A_{j} \frac{\partial}{\partial y_{j}}-C\right) u .
$$

Now consider the matrix

$$
M(\tau, \eta)=A_{0}^{-1}\left(\tau I-i \sum_{j=1}^{m} A_{j} \eta_{j}-C\right)
$$


where $\tau$ is a complex variable and $\eta_{j}(j=1, \cdots, m)$ are real. We put $\omega=\left\{(\tau, \eta) ; \operatorname{Re} \tau \geqslant M_{0}, \eta \in R^{m}\right\}$.

Lemma 1. The number of eigenvalue of $M(\tau, \eta)$ which have negative real part are invariant and coincide exactly with the number of negative eigenvalues of $A_{0}$, when $(\tau, \eta)$ is in $\omega$.

Proof. The number of eigenvalues with negative real part changes only when some of them become zero. Let $\tau^{0}$ and $\eta_{j}^{0}(j=1, \cdots, m)$ be such that $\operatorname{det}\left(M\left(\tau^{0}, \eta^{0}\right)-i \xi^{0} I\right)=0$, ( $\xi^{0}:$ real) where $\left(\tau^{0}, \eta^{0}\right)$ is in $\omega$. This equality is equivalent to:

$$
\operatorname{det}\left(\tau^{0} I-i \xi^{0} A_{0}-i \sum_{j=1}^{m} A_{j} \eta_{j}^{0}-C\right)=0 .
$$

But this is impossible because of hyperbolicity. Thus the number of eigenvalues with negative real part is invariant when $(\tau, \eta)$ is in $\omega$. We put $\eta_{j}=0(j=1, \cdots, m)$, then the characteristic equation takes the form

$$
\operatorname{det}(M(\tau, 0)-\xi I)=0
$$

and this is equivalent to

$$
\operatorname{det}\left(\tau I-\xi A_{0}-C\right)=0
$$

or

$$
\operatorname{det}\left(I-\frac{\xi}{\tau} A_{0}-\frac{1}{\tau} C\right)=0
$$

Giving $\tau$ only real value, we let $\tau \rightarrow+\infty$, then $\xi / \tau$ tends to the inverse of an eigenvalue of $A_{0}$. Accordingly, signs of $\operatorname{Re} \xi / \tau$ and signs of eigenvalues of $A_{0}$ coincide for large $\tau$. But as we have seen, the number of $\operatorname{Re} \xi / \tau$ which is negative is invariant when $(\tau, \eta)$ is in $\omega$. The lemma is thus proved.

By this lemma, we can define a linear subspace $E_{-}(\tau, \eta)$ (resp. $E_{+}(\tau, \eta)$ ) of $\mathbb{C}^{n}$ when $(\tau, \eta)$ is in $\omega$, which is spanned by all eigenvectors and generalized eigenvectors of $M(\tau, \eta)$ associated with those eigenvalues which have negative (resp. positive) real part. Evidently $E_{-}(\tau, \eta)$ is of $k$-dimensional. We call this a negative eigenspace (and also call the space $E_{+}(\tau, \eta)$ a positive eigenspace). 
3. Now we are ready to formulate our result.

Theorem. The mixed problem (1), (2) is weakly $L^{2}$-well-posed if and only if

$$
\mathbb{C}^{n}=\operatorname{Ker} B+E_{-}(\tau, \eta)
$$

for any $(\tau, \eta)$ in $\omega$.

(Here + means a direct sum of linear subspaces).

Proof. As it is convenient to handle the problem, we put the initial condition to be zero by substracting the solution of corresponding Cauchy's problem. We get then an inhomogeneous boundary condition:

$$
B u(t, 0, y)=g(t, y)
$$

where $g(t, y)$ as a vector in $\mathbb{C}^{n}$ belongs to $\operatorname{Range} B$, and as a function in $t$ grows at most exporentially as $t \rightarrow+\infty$. To solve the mixed problem (1) and (2), we transform the system by Fourier-Laplace transformation to a system of ordinary differential equation:

$$
\tau \hat{u}=A_{0} \frac{d \hat{u}}{d x}+i \sum_{j=1}^{m} A_{j} \eta_{j} \hat{u}+C \hat{u}
$$

that is,

$$
\frac{d \hat{u}}{d x}=A_{0}^{-1}\left(\tau I-i \sum_{j=1}^{m} A_{j} \eta_{j}-C\right) \hat{u}=M(\tau, \eta) \hat{u}
$$

where $\hat{u}=\hat{u}(\tau, x, \eta)$ satisfies an initial condition

$$
B \hat{u}(\tau, 0, \eta)=\hat{g}(\tau, \eta) .
$$

The general solution of (4) is

$$
\hat{u}(\tau, x, y)=e^{M(\tau, \eta) x} c(\tau, \eta)
$$

where the initial data $\hat{u}(\tau, 0, \eta)=c(\tau, \eta)$ comes from $(5)$ :

$$
B c(\tau, \eta)=\hat{g}(\tau, \eta) \text {. }
$$

Now we shall show that we must choose $c(\tau, \eta)$ from the space $E_{-}(\tau, \eta)$. Suppose that $c(\tau, \eta)$ does not belong to $E_{-}(\tau, \eta)$ for some $(\tau, \eta)$ in $\omega$, then the decomposition of the vector $c(\tau, \eta)$ into the sum

$$
c(\tau, \eta)=c_{+}(\tau, \eta)+c_{-}(\tau, \eta) \quad\left(c_{+} \in E_{+}, c_{-} \in E_{-}\right)
$$


shows that the solution (6) takes the form

$$
\hat{u}(\tau, x, y)=e^{M(\tau, \eta) x} c_{1}(\tau, \eta)+e^{v i(\tau, \eta) \tau} c_{-}(\tau, \eta)
$$

where the first term does not vanish for some $(\tau, \eta)$ in $\omega$. It is clear that this term increases exporeitially as $x \rightarrow+\infty$ and $u(\tau, x, \eta)$ fails to belong to any space $H^{m}\left(R_{+}^{1}\right)$.

Thus we have reacked two necessary conditions on $c(\tau, \eta)$ :

$$
\begin{aligned}
& B c(\tau, \eta)=\hat{g}(\tau, \eta) \in \text { Range } B, \\
& c(\tau, \eta) \in E_{-}(\tau, \eta) .
\end{aligned}
$$

In order to determire $c(\tau, \eta)$ from $\hat{g}(\tau, \eta)$ uniquely so that $c(\tau, \eta)$ belongs to $E_{-}(\tau, \eta)$, it is necessary and sufficient that two spaces $\operatorname{Ker} B$ and $E_{-}(\tau, \eta)$ satisfy the direct sum condition in the vector space $\mathbb{C}^{n}$ :

$$
\mathbb{C}^{n}=\operatorname{Ker} B+E_{-}(\tau, \eta) \text {. }
$$

We are now going to prove that this condition is sufficient in order trat the mixed problem is weakly $L^{2}$-wivell-posed. Since the condition (3) guarantees the urique solvability of $c(\tau, \eta)$ from $g(\tau, \eta)$, we can define a lirear operator $Q(\tau, \eta)$ from $\operatorname{Range} B$ onto $E_{-}(\tau, \eta)$ :

$$
c(\tau, \eta)=Q(\tau, \eta) \hat{g}(\tau, \eta)
$$

Then our "formal" solution $\hat{u}(\tau, x, \eta)$ is represented by

$$
\hat{u}(\tau, x, \eta)=e^{\pi / \tau, \eta) x} Q(\tau, \eta) \hat{g}(\tau, \eta) .
$$

Since $Q(\tau, \eta) \hat{g}(\tau, \eta)$ belongs to $\mathbb{E}_{-}(\tau, \eta)$, the solution (8) can be written as

$$
\hat{u}(\tau, x, \eta)=e^{M i(\tau, \eta) x} P_{-}(\tau, \eta) Q(\tau, \eta) \hat{g}(\tau, \eta)
$$

where $P_{-}(\tau, \eta)$ is the projector onto $\mathbb{E}_{-}(\tau, \eta)$ associated with the decomposition

$$
\mathbb{C}^{n}=E_{+}(\tau, \eta)+E_{-}(\tau, \eta)
$$

We divide the proof into several propositions.

Proposition 1. $e^{M(\tau, \eta) x} P_{-}(\tau, \eta)$ is holomorphic in the domain $\omega$.

Proof. This is almost obvious. Take the Cauchy integral form of the matrix, we have 


$$
e^{M(\tau, \eta) x} P_{-}(\tau, \eta)=\frac{1}{2 \pi i} \oint_{\Gamma(\tau, \eta)}(M(\tau, \eta)-\lambda I)^{-1} e^{\lambda x} d \lambda
$$

where the contour $\Gamma(\tau, \eta)$ is a closed curve in the domain $\operatorname{Re} \lambda<0$, surrounding all those eigenvalues of $M(\tau, \eta)$ which have negative real part. Although the curve $\Gamma$ depends on $(\tau, \eta)$, it is insensitive to a small change of $(\tau, \eta)$. Therefore Proposition 1 is valid.

Proposition 2. There exist a positive number $C$ and a rational number a such that the matrix norm is estimated as

$$
\left|e^{\pi(\tau, \eta) x} P_{-}(\tau, \eta)\right| \leqslant C\left(|\tau|^{2}+|\eta|^{2}\right)^{a}
$$

when $(\tau, \eta)$ belongs to $\omega$ and $|\tau|^{2}+|\eta|^{2} \rightarrow+\infty$.

Proof. Since $(M(\tau, \eta)-\lambda I)^{-1}=\frac{{ }^{t} \operatorname{Cof}(M(\tau, \eta)-\lambda I)}{\operatorname{det}(M(\tau, \eta)-\lambda I)}$, we have only to examine in the formula (9) the behavior of $\operatorname{det}(M(\tau, \eta)-\lambda I)$. Let $\lambda_{1}(\tau, \eta), \cdots, \lambda_{n}(\tau, \eta)$ be the eigenvalues of $M(\tau, \eta)$ (equal or not), and suppose that $\operatorname{Re} \lambda_{j}(\tau, \eta)<0 \quad(j=1, \cdots, k)$ and $\operatorname{Re} \lambda_{j}(\tau, \eta)>0 \quad(j=k+1$, $\cdots, n)$. We can choose the contour $\Gamma(\tau, \eta)$ so that for any $\lambda \in \Gamma(\tau, \eta)$

$$
\left|\lambda_{j}(\tau, \eta)-\lambda\right| \geqslant \frac{1}{3} \min _{1 \leqslant \nu \leqslant k}\left|\operatorname{Re} \lambda_{\nu}(\tau, \eta)\right| \quad(j=1, \cdots, n) .
$$

Then we have

$$
|\operatorname{det}(M(\tau, \eta)-\lambda I)|=\prod_{j=1}^{n}\left|\lambda_{j}(\tau, \eta)-\lambda\right| \geqslant \frac{1}{3^{n}}\left(\min _{1 \leqslant \nu \leqslant k}\left|\operatorname{Re} \lambda_{\nu}(\tau, \eta)\right|\right)^{n} .
$$

Therefore, if we show that there exist a positive constant $C$ and a rational number $a$ such that the inequality

$$
\min _{1 \leqslant \nu \leqslant k}\left|\operatorname{Re} \lambda_{\nu}(\tau, \eta)\right| \geqslant \frac{C}{\left(|\tau|^{2}+|\eta|^{2}\right)^{a}}
$$

holds, then (9) is estimated as

$$
\begin{aligned}
& \left|e^{M(\tau, \eta) x} P_{-}(\tau, \eta)\right| \\
\leqslant & \frac{1}{2 \pi} \sup _{\lambda \in \Gamma}\left|\frac{{ }^{t} \operatorname{Cof}(M(\tau, \eta)-\lambda I)}{\operatorname{det}(M(\tau, \eta)-\lambda I)}\right| \cdot\left|e^{\lambda x}\right| \cdot(\text { length of } \Gamma) \\
\leqslant & C \frac{\left(|\tau|^{2}+|\eta|^{2}\right)^{n}}{\left(\min _{1 \leqslant \nu \leqslant k}\left|\operatorname{Re} \lambda_{\nu}(\tau, \eta)\right|\right)^{n}} \\
\leqslant & C\left(|\tau|^{2}+|\eta|^{2}\right)^{n(1+a)} .
\end{aligned}
$$


Our aim is now the inequality (10) which is a consequence of Seidenberg-Tarski elimination theorem. Here we adopt Hörmander's formulation ([3] Lemma 2.1 of Appendix) in a slightly extended (but essentially equivalent) form.

Lemma 2. Let $Q(\sigma, \xi), R_{j}(\sigma, \xi)(j=1, \cdots, N)$ and $S_{k}(\sigma, \xi)(k=1$, $\cdots, L)$ be polynomials in $n+1$ real variables $\sigma, \xi=\left(\xi_{1}, \cdots, \xi_{n}\right)$, and suppose that $Q$ and $S_{k}$ have real coefficients. Assume that the set

$$
M(\sigma)=\left\{\xi ; R_{j}(\sigma, \xi)=0, S_{k}(\sigma, \xi) \leqslant 0(1 \leqslant j \leqslant N, 1 \leqslant k \leqslant L)\right\}
$$

is not empty for sufficiently large $\sigma$, then either

$$
\sup _{\xi \in M(\sigma)} Q(\sigma, \xi)=+\infty \quad \text { for all large } \sigma
$$

or else

$$
=C \sigma^{a}(1+o(1)) \text { as } \sigma \rightarrow+\infty,
$$

where $a$ is rational and $C$ is real.

Now, in the equation

$$
\operatorname{det}(M(\tau, \eta)-\lambda I)=\operatorname{det}(M(\tau, \eta)-(\operatorname{Re} \lambda+i \operatorname{Im} \lambda) I)=0
$$

we put $\frac{1}{\operatorname{Re} \lambda}=\rho$, then the equation is written as

$$
\operatorname{det}(\rho M(\tau, \eta)-(1+i \operatorname{Im} \lambda \cdot \rho) I)=0
$$

which is an algebraic equation in $m+4$ variables $\operatorname{Re} \tau, \operatorname{Im} \tau, \eta_{1}, \cdots, \eta_{m}$, $\rho$ and $\operatorname{Im} \lambda$. We consider moreover the inequality

$$
|\tau|^{2}+|\eta|^{2} \leqslant \sigma \quad \text { and } \quad \operatorname{Re} \tau \geqslant M_{0}
$$

and put

$$
M(\sigma)=\left\{\xi=\left(\operatorname{Re} \tau, \operatorname{Im} \tau, \eta_{1}, \cdots, \eta_{m}, \rho, \operatorname{Im} \lambda\right) ;(12) \text { and (13) are valid }\right\} .
$$

This set is evidently non-void for sufficiently large $\sigma$. So by Lemma 2 we have

$$
\sup _{\xi \in M(\sigma)}(-\rho)=+\infty \quad \text { or } \quad C^{\prime \prime}(1+o(1)) \quad \text { as } \sigma \rightarrow+\infty .
$$

But it is not $+\infty$, because on the compact domain (13), the real part $\operatorname{Re} \lambda$ of the solution of (11) is not equal to zero. Since 


$$
\sup _{\xi \in M(\sigma)}(-\rho)=\sup _{\substack{\left.|\tau|\right|^{2}+\left.|\eta|\right|^{2 \leqslant \sigma} \\ \operatorname{Re} \tau=M_{0}}} \max _{\substack{1 \leqslant \nu \leqslant k \\\left|\operatorname{Re} \lambda_{\nu}(\tau, \eta)\right|}},
$$

we have

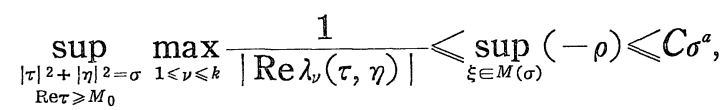

which means that in the domain $\omega$ there holds (10). Proposition 2 is thus proved.

Proposition 3. The matrix $Q(\tau, \eta)$ is holomorphic in $\omega$.

Proof. We calculate the explicit form of $Q(\tau, \eta)$. Since according to the condition (3) the rank of the constant matrix $B$ is $k$, we may assume without loss of generality that $B$ is a $k \times n$ matrix. We denote the row vectors of $B$ by $b_{1}, \cdots, b_{k}$. Now taking an arbitrary basis for $E_{-}(\tau, \eta)$, say, $v_{1}, \cdots, v_{k}$ (column vectors), the condition (3) can be written as

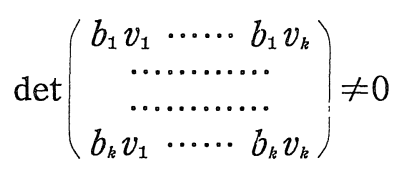

for any $(\tau, \eta)$ in $\omega$, and we have

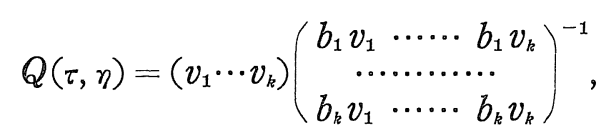

$\left(v_{1} \cdots v_{k}\right)$ being the $n \times k$ matrix whose column vectors are $v_{1}, \cdots, v_{k}$. We denote this matrix by $V$. Since $Q(\tau, \eta)$ is independent of the choice of the basis $v_{1}, \cdots, v_{k}$, we have only to show that for any fixed $\left(\tau^{0}, \eta^{0}\right)$ in $\omega$, there exists a neighborhood of $\left(\tau^{0}, \eta^{0}\right)$ such that for all $(\tau, \eta)$ in this neighborhood we can construct a basis for $E_{-}(\tau, \eta)$ whose components are holomorphic in $(\tau, \eta)$. Now take a fixed basis $v_{1}, \cdots, v_{k}$ for $E_{-}\left(\tau^{0}, \eta^{0}\right)$ and put again

$$
v_{j}(\tau, \eta)=P_{-}(\tau, \eta) v_{j}=\frac{1}{2 \pi i} \oint_{\Gamma}(M(\tau, \eta)-\lambda I)^{-1} v_{j} d \lambda
$$

where the contour $\Gamma$ in the domain $\operatorname{Re} \lambda<0$ is enclosing all eigenvalues of $M(\tau, \eta)$ which have negative real part. Clearly $v_{j}(\tau, \eta)(j=1, \cdots, k)$ 
is holomorphic in $(\tau, \eta)$ and $v_{j}\left(\tau^{0}, \eta^{0}\right)=P_{-}\left(\tau^{0}, \eta^{0}\right) v_{j}=v_{j}$, so that for sufficiently small change of values of $(\tau, \eta)$ near $\left(\tau^{0}, \eta^{0}\right)$ the vectors $v_{j}(\tau, \eta)$ are linearly independent. Proposition 3 is proved.

Proposition 4. There exist a positive constant $C$ and a rational number a such that for each element $q_{i j}(\tau, \eta)$ of the matrix $Q(\tau, \eta)$ we have

$$
\left|q_{i j}(\tau, \eta)\right| \leqslant C\left(|\tau|^{2}+|\eta|^{2}\right)^{a}
$$

when $(\tau, \eta)$ is in $\omega$ and $|\tau|^{2}+|\eta|^{2} \rightarrow \infty$.

Proof. We have seen that

$$
Q(\tau, \eta)=V(\tau, \eta)(B V(\tau, \eta))^{-1}
$$

We calculate this matrix more precisely. We have

$$
V(B V)^{-1}=\frac{1}{\operatorname{det}(B V)} \cdot V \cdot{ }^{t} \operatorname{Cof}(B V),
$$

and $(i, j)$-th element of the matrix $V^{t} \operatorname{Cof}(B V)$ takes the form

$$
\sum_{p=1}^{k} v_{i p} \Delta_{j p}=\operatorname{det}\left(\begin{array}{ccc}
b_{1} v_{1} \cdots \cdots & b_{1} v_{k} \\
\cdots \cdots \cdots & \cdots \\
v_{i 1} & \cdots \cdots & v_{i k} \\
b_{k} v_{1} & \cdots \cdots & b_{k} v_{k}
\end{array}\right)<j \text {-th row }
$$

where $v_{i j}$ is the $i$-th component of the vector $v_{j}$ and $\Delta_{i j}$ is $(j, i)$-th cofactor of $B V$. But the right side of this equality is equal to $\operatorname{det}\left(B_{i j} V\right)$ where $B_{i j}$ is the matrix obtained from $B$ by replacing the row vector $b_{j}$ by $e_{i}=(0 \cdots 1 \cdots 0)$ (all terms are 0 except $i$-th). Thus we have reached a very simple expression:

$$
q_{i j}(\tau, \eta)=\frac{\operatorname{det}\left(B_{i j} V\right)}{\operatorname{det}(B V)}
$$

From now on, we omit the suffix $i, j$ and put $B_{i j}=B_{1}$. Again we try to apply Lemma 2. Since $q(\tau, \eta)$ is holomorphic, it is sufficient to prove the inequality (14) in an open dense subset of $\omega$. We show that there exists an algebraic variety (whose complement is a connected, open and dense in $\omega$ ) except on which the inequality (14) is valid. 
We decompose the polynomial $\operatorname{det}(M(\tau, \eta)-\lambda I)$ into the product of irreducible factors:

$$
\operatorname{det}(M(\tau, \eta)-\lambda I)=p_{1}(\lambda, \tau, \eta)^{l_{1} \cdots} p_{\nu}(\lambda, \tau, \eta)^{l_{\nu}}
$$

and put

$$
P(\lambda, \tau, \eta)=p_{1}(\lambda, \tau, \eta) \cdots p_{\nu}(\lambda, \tau, \eta) .
$$

Then the resultant $R(\tau, \eta)$ of $P(\lambda, \tau, \eta)$ and $\frac{\partial P}{\partial \lambda}(\lambda, \tau, \eta)$ is not identically zero. Now consider the inequality

$$
R(\tau, \eta) \neq 0
$$

The complement of the algebraic variety $R(\tau, \eta)=0$ is dense, open and connected, since $\tau$ is a complex variable. On this set all roots of $P(\lambda, \tau, \eta)=0$ in $\lambda$ is distinct. Next, consider equations

$$
p_{1}(\lambda, \tau, \eta)=0, \cdots, p_{\nu}(\lambda, \tau, \eta)=0
$$

and inequalities

$$
\operatorname{Re} \lambda_{1}<0, \cdots, \operatorname{Re} \lambda_{\nu}<0 .
$$

Using these parameters we construct a basis $v_{1}, \cdots, v_{k}$ for $E_{-}(\tau, \eta)$ whose each component is a polynomial in $\lambda_{j}, \tau$ and $\eta$. For this purpose we recall the routine of obtaining generalized eigenvector. To do it, we put

$$
\left(M(\tau, \eta)-\lambda_{j} I\right)^{l_{j}} w=0
$$

If $\lambda_{j}$ is an eigenvalue of multiplicity $l_{j}$, the rank of the matrix $\left(M(\tau, \eta)-\lambda_{j} I\right)^{l_{j}}$ is exactly $n-l_{j}$, so we can solve the above simultaneous linear equation by Cramer's method and get solution vectors $w$. In this process, each component of $w$ can be chosen as being some $\left(n-l_{j}\right)$ minor of $\left(M(\tau, \eta)-\lambda_{j} I\right)^{l_{j}}$, and we make all possible combinations of this type. Among these combinations there may be many solutions consisting of only 0 components, but the process of computing each minor is entirely formal so that it is independent of whether the variable $\lambda_{j}$ is equal to some eigenvalue of $M(\tau, \eta)$ or not. The set of those polynomial vectors $w$ constructed by the above manner we denote by $W_{j}(j=1, \cdots, \nu)$. If the degree of $p_{j}$ in $\lambda$ is $d_{j}$, we can find $d_{j} \times l_{j}$ linearly independent vectors in $W_{j}$ when $\lambda_{j}$ is equal to root of $p_{j}\left(\lambda_{j}, \tau, \eta\right)$ 
$=0$. Choose arbitrar $y d_{j} \times l_{j}$ vectors from $W_{j}(j=1, \cdots, \nu)$ and construct the matrix $V$, and consider for each choice of these vectors the equation

$$
\operatorname{det}(B V)-q \cdot \operatorname{det}\left(B_{1} V\right)=0
$$

where $q$ is a free variable. Clearly $\operatorname{det}(B V) \neq 0$ if and only if chosen vectors are ail non-zero and linearly irdependent. But if $\operatorname{det}(B V)=0$, then also $\operatorname{det}\left(B_{1} V\right)=0$ simultaneously and so the equation (18) is trivially valid. If $\operatorname{det}(B V) \neq 0$, then the equation (18) is independent of the choice of the vectors in $W_{j}(j=1, \cdots, \nu)$. Thus, although there are so many viays of choice of vectors from $W_{j}$, constructed algebraic equation (18) in the variables $\left(\tau, \eta, \lambda_{1}, \cdots, \lambda_{\nu}, q\right)$ is only one.

In order to apply Lemma 2 , we put

$$
\begin{array}{r}
M(\sigma)=\left\{\xi=\left(\operatorname{Re} \tau, \operatorname{Im} \tau, \eta, \operatorname{Re} \lambda_{j}, \operatorname{Im} \lambda_{j}, \operatorname{Re} q, \operatorname{Im} q\right)\right. \\
(13),(15),(16),(17) \text { and }(18) \text { hold }\} .
\end{array}
$$

Clearly $M(\sigma)$ is non-void for sufficient large $\sigma$, so by Lemma 2 , we have

$$
\sup _{\xi \in M(\sigma)}|q|^{2}=+\infty \quad \text { or else } C \sigma^{a}(1+o(1)) \text { as } \sigma \rightarrow+\infty \text {. }
$$

$M(\sigma)$ being compact for finite $\sigma$, we have

$$
\sup _{\xi \in M(\sigma)}|q|^{2} \leqslant C \sigma^{a} \quad \text { as } \sigma \rightarrow+\infty
$$

from which it follows that

$$
\sup _{|\tau| 2+|\eta|^{2=\sigma}}|q|^{2} \leqslant \sup _{\xi \in M(\sigma)}|q|^{2} \leqslant C \sigma^{a}
$$

that is, on the complement of the variety $R(\tau, \eta)=0$ in $\omega$, we have

$$
\mid q(\tau, \eta) !^{2} \leqslant C\left(|\tau|^{2}+|\eta|^{2}\right)^{a} .
$$

This proves Proposition 4.

Thus the operator

$$
e^{M(\tau, \eta) x} Q(\tau, \eta): \hat{g}(\tau, \eta) \rightarrow \hat{u}(\tau, x, \eta)
$$

is holomorphic in $(\tau, \eta)$ in the domain $\omega$ and its norm is estimated as

$$
\left|e^{M(\tau, \eta) 2} Q(\tau, \eta)\right| \leqslant C\left(|\tau|^{2}+|\eta|^{2}\right)^{a}
$$

for some $C>0$ and $a$. Hence we can operate the inverse Fourier-Laplace transformation to the function $\hat{u}(\tau, x, \eta)$ in distribution sense (if 
$\hat{g}(\tau, \eta)$ is majorated by $\left(|\tau|^{2}+|\eta|^{2}\right)^{-b}$ with sufficiently large $b$, this transformation is performed in usual sense). Proof of our theorem is completed.

\section{References}

[1] Hersh, R., Mixed problem in several variables, J. Math. Mech. 12 (1963), 317-334.

[2] - Boundary conditions for equations of evolution, Arch. Rat. Mech. Anal. 16 (1964), 243-264.

[3] Hörmander, L., Linear Partial Differential Operators, Springer, 1963. 